\title{
MICROMECHANICAL INTERACTION MODEL ACCOUNTING FOR THE SPATIAL DISTRIBUTION OF INCLUSIONS IN ELASTIC-VISCOPLASTIC COMPOSITES
}

\author{
$\underline{\text { K. Kowalczyk-Gajewska }}{ }^{1 *}$, M. Majewski ${ }^{1}$, S. Mercier $^{2}$, A. Molinari ${ }^{2}$ \\ ${ }^{1}$ Institute of Fundamental Technological Research, Polish Academy of Sciences, \\ Pawińskiego 5B, 02-106 Warsaw, Poland, \\ ${ }^{2}$ Universite de Lorraine -CNRS - Arts et Metiers ParisTech, Laboratoire d'Etude des Microstructures et \\ de Mecanique des Materiaux, 7 rue Felix Savart, 57070 METZ - FRANCE \\ *kkowalcz@ippt.pan.pl
}

A cluster interaction model [1] was proposed to account for the spatial distribution and morphology of particles when estimating the effective properties of elastic and thermoelastic composites. In the present study, this approach is extended to elastic-viscoplastic composites. To this end, the tangent linearization of the non-linear viscoplastic law and the concept of additive interaction equation [2] are used. Although the extension is formulated for the nonlinear case, first applications are considered for linear viscoelastic composites with a single family of inclusions, a situation rich enough to evaluate the interest of the cluster interaction approach.

The results of the model are compared to numerical homogenization for periodic unit cells with two cubic configurations: regular cubic and body-centered cubic. As concerns material properties of inclusions, three cases were analysed: elastic inclusions, and next viscoelastic inclusions which are stiffer or softer than the matrix, respectively. Comparisons with Finite Element simulations have shown that accounting for particle-to-particle interactions enables to significantly improve the accuracy of estimates (both in terms of overall and per phase responses) with respect to schemes ignoring these interaction effects, for instance, the additive tangent Mori-Tanaka model [3].

Acknowledgement: The research was partially supported by the project No. 2016/23/B/ST8/03418 of the National Science Centre, Poland.

\section{References}

[1] A. Molinari and M. El Mouden (1996) The problem of elastic inclusions at finite concentration. International Journal of Solids and Structures, 33, 3131-3150.

[2] A. Molinari (2002) Averaging models for heterogeneous viscoplastic and elastic viscoplastic materials. Journal of Engineering Materials and Technology, Transactions of the ASME, 124, 62-70.

[3] C. Czarnota, K. Kowalczyk-Gajewska, A. Salahouelhadj, M. Martiny and S. Mercier (2015) Modeling of the cyclic behavior of elastic-viscoplastic composites by the additive tangent Mori-Tanaka approach and validation by finite element calculations. International Journal of Solids and Structures, 56-57, 96-117. 